\title{
Fisiopatologia e terapia do cão com sepse: revisão
}

\author{
Breno Curty Barbosa ${ }^{*}$, Fernanda dos Santos Alves², Suzane Lilian Beier ${ }^{3}$, Rafael \\ Resende Faleiros ${ }^{3}$, Patricia Maria Coletto Freitas ${ }^{3}$
}

Universidade Federal de Minas Gerais, Departamento de Clínica e Cirurgia, Belo Horizonte, MG, Brasil.

${ }^{1}$ Médico Veterinário, Mestrando em Ciência Animal

${ }^{2}$ Médico Veterinário, Doutorando em Ciência Animal

${ }^{3}$ Médico Veterinário, Docente Departamento de Clínica e Cirurgia

*Email para correspondências: brenocurty@hotmail.com

\begin{abstract}
RESUMO. Os quadros de sepse em animais vêm se apresentando de forma bastante rotineira, exigindo dos médicos veterinários cada vez mais conhecimento sobre o assunto, além de uma equipe preparada, para adoção de estratégias terapêuticas rápidas e precisas. A sepse cursa inicialmente com um quadro da síndrome da resposta inflamatória sistêmica (SIRS) gerada por um agente infeccioso, que em muitos casos evolui para a síndrome e disfunção de múltiplos órgãos, a qual sem intervenções adequadas originam elevadas taxas de mortalidade. Contudo, a sepse na medicina veterinária e na medicina humana é responsável por alta mortalidade em unidades de terapia intensiva. Porém, sem o conhecimento da sua fisiopatologia não se pode intervir adequadamente. Assim, objetivou-se com esta revisão descrever a fisiopatologia e o tratamento para cães com sepse.
\end{abstract}

Palavras chave: Cão, fisiopatologia, sepse

\section{Pathophysiology and therapy dog with sepsis: Review}

\begin{abstract}
Septic states in animals have become common and it demands the veterinarian greater knowledge about this issue, also a qualified team in order to be able to have quick and precise therapeutic decisions. Sepsis courses with systemic inflammatory response syndrome (SRIS), caused by and infectious agent, that most of times evolve into multiple organs disfunction syndrome (MODS) with high mortality rates. However, sepsis in veterinary medicine and in human medicine is responsible for high mortality rates in intensive care units. Nevertheless without the knowledge about its pathophysiology one may not intervene appropriately. Through this literature review the objective is to describe the pathophysiology and the treatment of dogs with sepsis.
\end{abstract}

Keywords: dogs, pathophysiology, sepsis

\section{Introdução}

A sepse é definida como a Síndrome da Resposta Inflamatória Sistêmica (SIRS) do hospedeiro a uma infecção, seja ela por vírus, fungos ou bactérias (Theobaldo, 2012).

$\mathrm{Na}$ sepse grave observa-se um quadro de sepse associado com uma disfunção orgânica com alterações de perfusão. Dentre as disfunções orgânicas encontram-se uma diminuição da pressão arterial sistólica do valor basal, oligúria, elevação dos valores de lactato sanguíneo, alterações em nível de consciência; alterações em enzimas hepáticas e/ou renais; entre outros (Theobaldo, 2012).

A sepse grave tem sido diagnosticada com mais frequência na medicina veterinária e é responsável por alta mortalidade em unidades de terapia intensiva e em clínicas veterinárias, sendo de grande importância intervenções imediatas para seu controle e tratamento. Porém, sem o conhecimento da sua fisiopatologia não se pode intervir adequadamente. Assim, objetivou-se com esta revisão descrever a fisiopatologia e o tratamento para cães com sepse. 


\section{Revisão de literatura}

\section{Fisiopatologia da sepse}

A sepse representa uma resposta sistêmica do sistema imunológico a um agente infeccioso, na qual a virulência do patógeno e a resistência do hospedeiro regulam as repercussões da resposta inflamatória. Do patógeno, a virulência depende do número, tipo e local de organismos invasores, enquanto a imuno competência do hospedeiro é determinada por múltiplos fatores, dentre eles idade, sexo, predisposição genética, estado nutricional, medicamentos e condições subjacentes, como câncer. Uma maciça carga bacteriana pode suprimir os mecanismos inibitórios para controle da inflamação, levando à inflamação sistêmica (Salomão et al., 2014).

Assim, diversos estímulos mecânicos ou químicos, agindo na célula endotelial, aumentam a produção da enzima sintase do óxido nítrico (NOs), que tem por função catalisar a conversão de L-arginina e oxigênio para citrulina e óxido nítrico. Extremamente difusível, o óxido nítrico (NO) é inativado pela hemoglobina, com formação de meta-hemoglobina e, quando se difunde para as células do músculo liso adjacente às células endoteliais, propicia a ação da guanilato-ciclase, com aumento do GMP cíclico intra-citosólico nessas células. O GMP cíclico promove o menor acoplamento actino-miosina através da retirada do cálico intracelular. A consequência é o relaxamento da musculatura lisa e consequente vasodilatação. Além disso, a NOs induzida leva a uma produção intensa e desregulada de NO, que resulta em vasodilatação arteriolar e redução da resistência vascular periférica (Caldeira Filho \& Westphal, 2010).

Também na sepse ocorre uma resposta imune aguda e uma excessiva produção de citocinas pró-inflamatórias. As citocinas são mediadores proteicos de baixo peso molecular produzidos precoce e continuamente durante todo o episódio séptico (Lopes et al., 2010) e de acordo com Rau et al. (2007), a SRIS e a sepse são caracterizadas pela ativação de citocinas. As citocinas próinflamatórias, fator de necrose tumoral alfa (TNF a) e interleucina (IL-1) ativam células-alvo e induzem a produção de mediadores próinflamatórios, como outras citocinas, quimiocinas, espécies reativos de oxigênio (EROs), espécies reativas de nitrogênio (ERN), eicosanoides e enzimas proteolíticas (Salomão et al., 2014). As primeiras citocinas produzidas são o TNF $\boldsymbol{\alpha}$, interleucina (IL)-1 $\beta$ e a IL-6 (Lopes et al., 2010). A citocina pró-inflamatória 6 (IL-6) tem sua concentração significantemente elevada no plasma de cães com sepse, e elevados níveis de IL-6 à admissão deste paciente na terapia intensiva mostraram-se significativamente correlacionados à severidade da doença, ao aumento da mortalidade (Rau et al., 2007). A IL8 , in vivo, é um importante regulador da ativação e migração de neutrófilos, além de ser detectada no sangue de pacientes em sepse. A IL-10, por sua vez, faz parte da síndrome resposta anti inflamatória compensatória (CARS), estando também presente na circulação de pacientes sépticos (Thijs \& Hack, 1995). A CARS tem sido vista como um regulador da inflamação exacerbada e parece ser responsável pelo óbito em sepse severa (Andaluz-Odeja et al., 2012). Além disso, vários mediadores pró-inflamatórios associam-se ao recrutamento de neutrófilos, sendo um evento-chave para controle do foco infeccioso. Porém, a falência da migração neutrofílica para o foco infeccioso compromete diversas etapas da migração celular, incluindo a dessensibilização e internalização de receptores de fatores quimiotáxicos, redução da sinalização intracelular envolvida na quimiotaxia e redução do rolamento e adesão dos neutrófilos ao endotélio vascular, resultando no aumento da disseminação bacteriana (Salomão et al., 2014).

Outro evento que ocorre na sepse é o aumento dos valores de lactato. Segundo Stenvenson et al. (2007), isso ocorre devido ao desequilíbrio entre a produção e o consumo do lactato, causado por aumento da glicólise em condições de anaerobiose, ou em caso de perfusão sanguínea reduzida, o que leva à uma condição de anaerobiose. Assim, o lactato é um indicador para avaliação do metabolismo celular, para detectar a hipoperfusão e a resposta à terapia. Em um estudo realizado com cães acometidos por diversas doenças, determinou-se que $o$ prognóstico é pior para animais nos quais não houve queda no valor do lactato 6 horas após a admissão. Entretanto, sua efetividade como prognóstico se dá quando ocorre análise seriada deste parâmetro (Stenvenson et al., 2007). Entretanto, também pode-se observar na sepse grave a hipóxia citopática, na qual há oxigênio disponível, mas a célula não respira devido a um bloqueio mitocondrial, causado tanto pelas citocinas quanto pelo NO (Caldeira Filho \& Westphal, 2010). O óxido nítrico, o monóxido de carbono e o sulfito de hidrogênio são alguns dos principais mediadores pró-inflamatórios que 
podem inibir a função mitocondrial, competindo com o oxigênio. Além da competição com o oxigênio, estas moléculas podem levar a dano na estrutura proteica dos complexos transportadores de elétrons, provocando aumento da produção de espécies reativas de nitrogênio, como peroxinitrito e o radical superóxido. Além disso, outros processos concomitantes podem acentuar a disfunção mitocondrial, como a hiperglicemia e a ativação de vias de sinalização intracelulares ativadas pela resposta inflamatória. Todos esses mecanismos resultam na redução na produção celular de ATP. Se há manutenção do metabolismo normal celular, haverá redução dos níveis de ATP e consequente prejuízo à sua funcionalidade ou ativação de vias de morte celular (normalmente apoptose). Entretanto, pode ocorrer também redução da atividade celular, o que também levaria à redução de sua funcionalidade. Clinicamente isto se manifestará como redução das funções fisiológicas dos órgãos e, em modelos animais, a falência dos órgãos e deterioração clínica associada à disfunção mitocondrial e redução na taxa metabólica (Salomão et al., 2014).

Somada à hipóxia citopática, as alterações microvasculares também contribuem para insuficiência dos órgãos. Durante a sepse, diversos componentes da microcirculação são afetados e resultam em incompatibilidade entre oferta e o consumo de oxigênio. Entre as alterações estão a reduzida sensibilidade das células endoteliais a agentes vasoativos, a redução da deformabilidade das hemácias, a ativação de um estado pró-coagulante (formação de microtrombos e diminuição do fluxo tissular) e, por fim, a ativação leucocitária que leva à formação de neutrophil extracelullar traps que também estão relacionadas à redução do fluxo sanguíneo e disfunção orgânica. Não obstante, há grande heterogeneidade na distribuição do fluxo sanguíneo, com áreas de fluxo aumentado, de fluxo normal e de fluxo reduzido (Salomão et al., 2014).

$\mathrm{Na}$ sepse, a insuficiência bioenergética parece desempenhar importância central na patogênese da Síndrome da Disfunção de Múltiplos Órgãos (SDMO). A SDMO é marcada por relativa ausência de morte celular, o que sugere que a insuficiência bioenergética tem como consequência alterações na homeostase celular, que culminam com alteração de processos essenciais ao funcionamento celular.
Também, o estresse oxidativo, resultado de uma resposta inflamatória inerente à sepse, inicia mudanças na função mitocondrial que podem resultar em dano orgânico. É resultado de um desequilíbrio entre a produção de espécies reativas ao oxigênio (ROS) e os mecanismos de proteção por antioxidantes. Normalmente, um complexo sistema de antioxidantes é capaz de combater o estresse oxidativo e prevenir danos à mitocôndria. Esse complexo é constituído por caminhos enzimáticos e não enzimáticos, incluindo os sistemas superóxido dismutase magnesiana (MnSOD), glutationa (GSH) e tioredoxina (TSH), entre outros. Pela importância do estresse mediado por oxidantes, sendo seu dano mitocondrial fundamental na patologia da sepse, sugere-se que os antioxidantes possuam função terapêutica (Galley, 2011). A hipótese envolvendo o estresse oxidativo na sepse descreve a infecção como fator desencadeador do aumento da produção de ROS e de espécies reativas ao nitrogênio (RNS) concomitantemente ao declínio das defesas antioxidantes, resultando em dano celular, danos ao tônus vasomotor, disfunção miocárdica, injúria renal aguda e lesões em outros órgãos que culminarão em choque e posterior síndrome da disfunção de múltiplos órgãos (Vondessauer et al., 2011).

Em geral, a trombose intravascular, desencadeada pela inflamação local, é benéfica por dificultar a disseminação da infecção e da inflamação para o restante do organismo. Entretanto, a ativação incontrolável da coagulação é um dos marcos da sepse severa e, provavelmente, um dos principais motores na gênese da disfunção múltipla dos órgãos. A ativação ocorre através da via extrínseca da coagulação, com grandes quantidades de fator tissular (FT) expressas por células endoteliais e monócitos, parecendo depender principalmente da IL-6. O aumento da atividade coagulante não é suficientemente contrabalançado pelos inibidores naturais da coagulação na sepse grave, que se constituem da proteína C ativada (PCA), antitrombina III (AT) e o inibidor da via do fator tissular (TFPI). Na sepse grave, os níveis de PCA e AT estão diminuídos por resultado de um elevado consumo e degradação e de síntese inadequada (Caldeira Filho \& Westphal, 2010).

\section{Sepse e as disfunções orgânicas}

$\mathrm{Na}$ sepse, o acometimento de diferentes sistemas orgânicos culmina em complicações e pior prognóstico para os pacientes. 
O perfil hemodinâmico clássico do choque séptico é a hipotensão refratária a volume, intensa redução da resistência vascular periférica (RVS) e elevação do débito cardíaco (DC). A concomitância de hipovolemia, vasodilatação, disfunção miocárdica e da obstrução microvascular gerada por microtrombos implica em isquemia e hipoperfusão. A normalização das variáveis mecânicas (débito cardíaco, pré-carga, pós-carga, volume sistólico e outros) deve resultar na manutenção do transporte e na adequação da relação entre oferta e consumo de oxigênio de acordo com a demanda orgânica (Salomão et al., 2014).

A disfunção miocárdica observada na sepse parece não ser causada por hipoperfusão miocárdica. Nas fases iniciais, a produção de citocinas induz alterações no funcionamento do miocárdio e na produção de NO. Esse mediador altera a sensibilidade dos miofilamentos de cálcio, a resposta das vias adrenérgicas intracelulares e a função mitocondrial. Ocorrem também alterações independentes do NO que geram alterações na contratilidade. A disfunção miocárdica caracteriza-se por ser reversível, o que demonstra que a lesão definitiva por apoptose ou necrose miocárdica parece não ter grande significado (Salomão et al., 2014). No homem, um estudo observou que os sobreviventes apresentavam dilatação aguda do ventrículo esquerdo (VE), com aumento no volume sistólico e diastólico do VE, apesar da redução da fração de ejeção do ventrículo esquerdo (FEVE) para valores menores que $40 \%$. Acredita-se que tal alteração seja devido a uma adaptação aguda do coração em uma tentativa de manter o débito cardíaco, e essa adaptação é reversível à medida que há resolução do quadro séptico (Parker et al., 1984).

O acometimento pulmonar foi observado em $79 \%$ dos pacientes humanos em um estudo epidemiológico, com observação de diversos fatores de ocorrência simultânea, como o ingresso de neutrófilos na região alveolocapilar, edema intersticial, perda de surfactante e exsudatos fibrinosos alveolares (Caldeira Filho \& Westphal, 2010). Sabe-se que o pulmão é um órgão que frequentemente evolui com disfunção na sepse, mesmo que o foco infeccioso não seja pulmonar. A Síndrome da Angústia Respiratória Aguda (SARA) é uma complicação comum e grave em pacientes com sepse grave, e a presença de mediadores inflamatórios na circulação sistêmica pode ser o responsável pela disfunção orgânica que acompanha o desenvolvimento dessa síndrome. Durante a sepse, a barreira alveolocapilar pode estar lesada, permitindo que as citocinas e outras proteínas cheguem ao alvéolo. A IL-8 é a citocina mais importante no recrutamento de células polimorfonucleares para o espaço alveolar, causando dano endotelial. Tal fato resulta em aumento de permeabilidade, causando aumento do influxo de proteínas plasmáticas e células que levam à inativação do surfactante pulmonar (Kitsis, 2010).

O território esplâncnico é o primeiro a sofrer com a hipoperfusão e o último a recuperá-la. Dentre as vísceras, o intestino é extremamente susceptível à redução da perfusão e oxigenação, e assim a lesão isquêmica do intestino delgado desenvolve-se rapidamente. São diversas as repercussões do hipofluxo esplâncnico: comprometimento da viabilidade de anastomoses, aumento de permeabilidade dos capilares da mucosa intestinal e translocação bacteriana (Caldeira Filho \& Westphal, 2010). Assim, a circulação esplâncnica é uma das primeiras a ser comprometida em situações em que ocorrem redução do índice cardíaco. Essa circulação é normalizada apenas tardiamente, após o restabelecimento hemodinâmico. A maior concentração de células linfóides é encontrada no sistema gastrointestinal e sua ativação pode inclusive estar relacionada à perpetuação da resposta inflamatória. Em humanos, a abordagem da disfunção gastrointestinal aguda (DGIA) é realizada pelo reconhecimento e tratamento de sinais gastrointestinais relacionados à doença aguda. A disfunção e perda de enterócitos, alterações do trânsito intestinal (constipação ou diarréia), alteração da motilidade intestinal e retardo na progressão da nutrição enteral, distensão abdominal e hipertensão intraabdominal e sangramento digestivo são algumas das manifestações da DGIA (Azevedo, 2014).

A injúria renal aguda (IRA) relacionada à sepse é muito comum e determina elevada mortalidade quando se comparam pacientes sépticos com ou sem IRA. É a causa principal ou fator adjuvante em até $50 \%$ dos casos novos de IRA que ocorrem no paciente humano crítico (Vieira Júnior, 2014). Os mecanismos fisiopatológicos que auxiliam a explicar a IRA da sepse podem ser divididos em mecanismos hemodinâmicos sistêmicos e regionais, e em mecanismos não hemodinâmicos. A hipovolemia relativa ou o choque possuem grande importância ao determinarem redução do fluxo renal e 
disfunção orgânica. Ressalta-se que mesmo na ausência de choque manifesto, pacientes hipertensos e idosos, principalmente aqueles com doença renal crônica, são susceptíveis a níveis de PAM considerados aceitáveis devido à perda de auto-regulação intra-renal. Entretanto, os fatores hemodinâmicos isoladamente não explicam por completo o desenvolvimento da IRA. Outros mecanismos presentes na sepse, que incluem ativação da cascata inflamatória sistêmica e intrarenal, estresse oxidativo sistêmico e intra-renal, ativação do processo de apoptose renal e alterações da microcirculação intra-renal com hipóxia tecidual, podem explicar parte da fisiopatologia da IRA na sepse (Vieira Júnior, 2014).

\section{Manejo do paciente com sepse}

A campanha Sobrevivendo à Sepse (Surviving Sepsis Campaign) de 2012 trouxe recomendações para o tratamento da sepse grave e do choque séptico, por meio de um consenso entre 68 especialistas internacionais, na intenção de proporcionar orientações aos clínicos (Dellinger et al., 2013). Assim, algumas metas foram traçadas para guiar a terapia no homem e nos animais com quadro clínico de sepse grave. Essas metas consistem na manutenção de pressão arterial média (PAM) maior que $65 \mathrm{mmHg}$, débito urinário maior que $0,5 \mathrm{~mL} / \mathrm{kg} / \mathrm{h}$, pressão venosa central entre 8 e $12 \mathrm{cmH}_{2} \mathrm{O}$, saturação venosa de veia cava superior maior que $70 \%$ ou saturação venosa mista maior que $65 \%$, bem como a normalização do lactato sérico ou sua queda maior ou igual a $20 \%$ do valor inicial após as duas primeiras horas de terapia (Dellinger et al., 2013).

Assim, o manejo hemodinâmico em sepse grave e choque séptico incluem a rápida restauração do volume intravascular e equilíbrio adequado entre a oferta sistêmica de oxigênio e sua demanda (Oliveira et al., 2002). Lopes et al. (2010) e Dellinger et al. (2013) recomendaram que a restituição volêmica deve ser realizada de forma precoce e durante as 6 horas iniciais do tratamento, com o acompanhamento dos seguintes parâmetros: pressão venosa central que deve oscilar entre 8 a $12 \mathrm{mmHg}$, pressão arterial média maior ou igual a $65 \mathrm{mmHg}$, débito urinário maior ou igual a $0,5 \mathrm{~mL} / \mathrm{kg} / \mathrm{h}$ e saturação venosa central ou mista de $70 \%$ ou $65 \%$, respectivamente, além da normalização dos níveis de lactato em pacientes com hiperlactatemia. Rivers et al. (2001) demonstraram que a terapia precoce guiada por metas reduziu a incidência de falência múltipla dos órgãos e mortalidade, por meio da manipulação da pré-carga, pós-carga e contratilidade cardíaca. A infusão de grande quantidade de fluidos é usualmente necessária para normalizar a pré-carga e pressão de enchimento, com o objetivo de restabelecer perfusão tecidual adequada e a oferta de oxigênio. Entretanto, essa infusão pode resultar em edema pulmonar e de outros tecidos, além de que a repleção de volume com normalização hemodinâmica podem não ser suficientes para prevenir a disfunção microcirculatória. Ressaltase que a restituição hemodinâmica per se pode reduzir a resposta inflamatória na sepse, reduzindo o fenômeno de isquemia e reperfusão. Apesar de a reposição volêmica precoce ser iniciada por meio da expansão intravascular por meio de soluções cristalóides ou colóides, o uso de drogas vasoativas e inotrópicas pode ser necessário (Lopes et al., 2010). A disfunção vascular relacionada à sepse é multifatorial e pode necessitar de altas doses de vasopressores (Ramos \& Azevedo, 2014). A norepinefrina é o vasopressor de escolha (Dellinger et al., 2013; Ramos \& Azevedo, 2014) e pode ser potencializada por associação com epinefrina ou vasopressina (Dellinger et al., 2013). A dopamina pode ser utilizada em pacientes com baixo risco de desenvolvimento de taquiarritmias e bradicardia. A droga inotrópica de escolha é a dobutamina, indicada na presença de disfunção miocárdica ou de sinais de hipoperfusão apesar da restauração adequada do volume intravascular e da pressão arterial média (Dellinger et al., 2013). O seu uso precoce mostrou-se uma importante estratégia para reversão da hipoperfusão antes da progressão das disfunções orgânicas e da SDMO (Síndrome e disfunção de multiplos órgãos) (Rivers et al., 2001). Não deve ser utilizado, entretanto, visando alcançar a supranormalização das variáveis hemodinâmicas de forma tardia (Ramos \& Azevedo, 2014).

Além dessas medidas precoces descritas acima, deve-se realizar cultura apropriada e hemocultura em pelo menos dois sítios no paciente. Entretanto, esses procedimentos não podem durar mais que 45 minutos da admissão do paciente com sepse, pois antimicrobianos devem ser administrados o mais precoce possível. Recomenda-se o uso combinado de um betalactâmico associado a um aminoglicosídeo ou a uma fluorquinolona ou a um macrolídeo, 
mantendo-se essa terapia empírica por no máximo 3 a 5 dias. Rabelo (2013) sugeriu o uso de cefalotina como antibiótico empírico inicial, associada ou não a uma quinolona, e ainda recomendou que o fármaco escolhido para uso na sala de emergência em casos de sepse grave ou choque séptico não sejam utilizados na rotina de internamento e mesmo em consultas para tratamentos de rotina. Contudo, o espectro deve ser reduzido precocemente, de acordo com os resultados do antibiograma, e recomenda-se que o tempo de terapia seja de 7 a 10 dias, prolongando-se apenas em caso de paciente com resposta clínica lenta, foco infeccioso não drenável, bacteremia com Staphylococcus aureus e infecções fúngicas ou virais, bem como deficiências imunológicas. Ressalta-se que antimicrobianos não devem ser utilizados em pacientes em estado inflamatório grave de causa não infecciosa (Dellinger et al., 2013). Após 48 a 72 horas, de acordo com Lopes et al. (2010), a terapêutica deve ser reavaliada com base em dados clínicos e microbiológicos, que podem indicar manutenção do esquema inicial, descalonamento, ampliação do espectro ou mesmo sua descontinuação. Além disso, pode-se ser necessário ampliar o espectro antimicrobiano, caso haja risco de bactérias resistentes, que pode ser estratificado por dados como uso recente de antibióticos, tempo de internação prolongado, imunossupressão e presença de dispositivos invasivos como cateter venoso central, sonda vesical de demora, tubo orotraqueal, entre outros (Lopes et al., 2010). Vale ressaltar que quando há atraso em iniciar a antibioticoterapia, ou a escolha do antibiótico é direcionado a uma classe específica de micro-organismo, ou quando o agente é resistente ao fármaco escolhido, pode-se ocorrer falha na terapia com antibióticos (Lopes et al., 2010). Além disso, alguns aspectos hemodinâmicos podem levar à subconcentração nos níveis de antimicrobianos nos tecidos. São exemplos disso o aumento do débito cardíaco (DC), que pode causar um aumento do clearance das drogas; o aumento da perfusão tecidual em órgãos responsáveis pela eliminação e ou metabolismo, causando alterações tanto no clearance quanto na distribuição e a hipoperfusão tecidual, comprometendo desta forma a penetração do antimicrobiano no sítio de infecção (Lisboa \& Nagel, 2014).

Outros fármacos que podem ser necessários em pacientes com sepse são os corticóides. Neste quadro sua produção pode estar insuficiente ou até mesmo elevada por liberação aumentada de hormônio adrenocorticotrófico (ACTH) pela hipófise, porém sua função pode estar diminuída devido à resistência periférica aos receptores. É possível ainda que mesmo níveis elevados não sejam adequado à demanda metabólica do paciente. Contudo, as recomendações da Campanha de Sobrevivência à Sepse orientam que a hidrocortisona seja usada apenas em pacientes em choque séptico refratário, na dose de $0,5 \mathrm{mg} / \mathrm{kg}$ a cada 6 horas durante 3 dias, espaçando para a cada 12 horas por mais 2 dias. Outra opção é a utilização sob forma de infusão contínua, na taxa de $0,08 \mathrm{mg} / \mathrm{kg} / \mathrm{h}$ (Creedon, 2015). A recomendação ressalta que corticóide não deve ser utilizado em paciente sem choque, exceto se seu uso era crônico ou em caso de razão para sua utilização (Dellinger et al., 2013; Coimbra \& Machado, 2014). A Campanha ainda recomenda que a dose deste fármaco seja reduzido gradualmente assim que os vasoativos não sejam mais necessários (Dellinger et al., 2013).

Além da terapia inicial descrita acima, mais alguns fatores são importantes no manejo do paciente com sepse. Entre eles o controle glicêmico, pois a hiperglicemia tem participação nos processos de dano celular, resposta imune, funcionamento da microvasculatura, aumento na produção de citocinas, alterações estruturais em organelas celulares como a mitocôndria, além de associar-se a um estado de hipercoagulabilidade (Coimbra \& Machado, 2014). Assim, seu controle demonstrou redução de infecções de corrente sanguínea, do estado inflamatório, insuficiência renal com necessidade de terapia de substituição renal, polineuropatia, dias de ventilação mecânica e de hemotranfusões (Lopes et al., 2010). Para tal, deve-se utilizar a insulina, que além de controlar a hiperglicemia, reduz a resistência à insulina, promove vasodilatação, aumenta a captação de glicose por tecidos isquêmicos e possui propriedades antiinflamatórias (Coimbra \& Machado, 2014). Sugere-se que a insulinoterapia seja tratada sempre que a glicemia ultrapassar $180 \mathrm{mg} / \mathrm{dL} \mathrm{em}$ cães ou $250 \mathrm{mg} / \mathrm{dL}$ em gatos, por meio de infusão contínua de insulina regular iniciada a $0,05 \mathrm{UI} / \mathrm{kg} / \mathrm{h}$. Deve haver monitoração contínua dos níveis glicêmicos e de potássio para evitar iatrogenias (Kloss Filho \& Rabelo, 2013).

Além do controle da glicemia, o suporte nutricional deve ser instituído. Sugere-se que se inicie após 48 horas de restabelecimento de 
perfusão sanguínea, por via enteral ou oral. A nutrição plena também não é recomendada na primeira semana, optando-se pela nutrição trófica e avançando-se apenas se ocorrer tolerância adequada. A nutrição parenteral isolada ou em suplementação à dieta enteral não deve ser utilizada nos primeiros sete dias após o diagnóstico, bem como não se recomenda o uso de nutrição suplementar moduladora (Dellinger et al., 2013; Coimbra \& Machado, 2014).

A transfusão de componentes sanguíneos deve ser considerada quando a hemoglobina estiver menor que $7 \mathrm{~g} / \mathrm{dL}$, apenas após resolução da hipoperfusão e na ausência de circunstâncias como isquemia miocárdica, hipoxemia grave, entre outras, visando um valor de 7 a $9 \mathrm{mg} / \mathrm{dL}$ de hemoglobina em adultos. A transfusão de células vermelhas aumenta a $\mathrm{DO}_{2}$ em pacientes sépticos, mas não aumenta a $\mathrm{VO}_{2}$ usualmente (Dellinger et al., 2013).

Outras recomendações da Campanha incluem o uso de ventilação mecânica em pacientes com SARA, a correção de distúrbios ácido-básicos e eletrolíticos, a profilaxia para trombose venosa profunda, a profilaxia para úlceras gástricas e de úlceras de pressão (Dellinger et al., 2013).

Assim, pode-se concluir que a sepse cursa com várias alterações na fisiologia do animal, sendo de grande importância o conhecimento da sua fisiopatologia, e também intervenções imediatas para seu controle e tratamento.

\section{Referências Bibliográficas}

Andaluz-Oleja, D., Bobillo, F., Iglesias, V., Almansa, R., Rico, L., Gandía, F., Resino, S., Tamayo, E., Lejarazu, R. O. \& BermejoMartin, J. F. (2012). A combined score of proand anti-inflammatory interleukins improves mortality prediction in severe sepsis. Cytokine, 57, 332-336.

Azevedo, R. 2014. Disfunção gastrointestinal aguda na sepse. In.: Azevedo, L.C.P. \& Machado, F.R. (Ed.). Sepse. São Paulo, Atheneu, 13-20.

Caldeira Filho, M. \& Westphal, G. A. (2010). Fisiopatologia da sepse e do choque séptico. In.: Guimarães, H.P., Lopes, R.D. \& Lopes, A.C. Tratado de medicina de urgência, emergência, pronto-socorro e UTI. São Paulo, Atheneu, 2,1211- 1225.

Coimbra, K.T.F. \& Machado, F. R. (2014). Terapias adjuvantes da sepse. In.: Azevedo,
L.C.P. \& Machado, F.R. (Ed.). Sepse. São Paulo, Atheneu, 13-20.

Creedon, J. M. B. (2015). Controversies surrounding critical illness-related corticosteroid insufficiency in animals. Journal of Veterinary Emergency and Critical Care, 25:107-112.

Dellinger, R.P., Levy, M.M., Rhodes A., Annane, D., Gerlack, H., Opal, S. M., Sevransky, J. E., Sprung, C. L., Douglas, I. S., Jaeschke, R., Osborn, T. M., Nunnally, M. E., Townsend, S. R., Reinhart, K., Angus, D. C., Deutschman, S., Machado, F. R., Rubenfeld, G. D., Webb, S., Beale, R. J., Vincent, J. \& Moreno, R. (2013). Surviving Sepsis Campaign: International Guidelines for management of severe sepsis and septic shock: 2013. Critical Care Medicine, 41(2):183-194.

Galey, H.F. (2011). Oxidative stress and mitochondrial dysfunction in sepsis. British Journal of Anaesthesia, 1(107):57-64.

Kitsis, M. (2010). Avaliação de complicações pulmonares em cães com sepse grave submetidos à terapia intensiva. Dissertação. (Mestrado em ciência). Faculdade de Medicina da Universidade de São Paulo, São Paulo, f.94.

Kloss Filho, J.C. \& Rabelo, R.C. (2013). Controle glicêmico. In.: Rabelo, R (Ed.). Emergências de pequenos animais - Condutas clínicas e cirúrgicas no paciente grave. Rio de Janeiro: Elsevier, 316-322.

Lisboa, T. \& Nagel, F. (2014). Terapia antimicrobiana na sepse. In.: Azevedo, L.C.P. \& Machado, F.R. (Ed.). Sepse. São Paulo, Atheneu, 13-20.

Lopes, R.D., Silva, A.M.B. \& Bonillha, A.M.M. (2010). Sepse grave e choque séptico: aspectos clínicos. In.: Guimarães, H.P., Lopes, R.D. \& Lopes, A.C. Tratado de medicina de urgência, emergência, pronto-socorro e UTI. São Paulo, Atheneu, 2:1227-1238.

Oliveira, R. P., Velasco, I., Soriano, F. G. \& Friedman, G. (2002). Clinical review: Hypertonic saline resuscitation in sepsis. Critical Care, 5(6):418-423.

Parker, M.M., Shelhamer, J.H., Bacharach S. L., Green, M. S., Natanson, M. D. C., Terri, M., Frederick, B. S. N., Damsk, R. N. \& Parrillo, J. E. (1984). Profund but reversible myocardial depression in patients with septic 
shock. Annals Internal Medicine, 100:483490.

Rabelo, R.C. (2013). Sepse, sepse grave e choque séptico. In.: Rabelo, R. (Ed.) Emergências de pequenos animais - Condutas clínicas e cirúrgicas no paciente grave. Rio de Janeiro, Elsevier, 323-340.

Ramos, F.J.S. \& Azevedo, L.C.P. (2014). Suporte hemodinâmico no choque sépticos. In.: Azevedo, L.C.P. \& Machado, F.R. (Ed.). Sepse. São Paulo, Atheneu, 13-20.

Rau, S., Kohn, B., Richter, C. Fensk, N., Kuchenhoff, H., Hartmann, K., Hartle, S., Kaspers, B. \& Hirschberger, J. (2007). Plasma interleukin-6 response is predictive for severity and mortality in canine systemic inflammatory response syndrome and sepsis. Veterinary Clinical Pathology, 36(3):253-260.

Rivers, E., Nguyen, B., Havstad, S., Ressler, J. B. S., Muzzin, A. B. S., Knoblich, B. M. D., Peterson, E. \& Tomlanovich, M. (2001). Early goal-directed therapy in the treatment of severe sepsis and septic shock. The New England Journal of Medicine, 345(19):13681377.

Salomão, R., Petronilho, F. \& Ritter, C. (2014). Fisiopatologia da Sepse. In.: Azevedo, L.C.P. \& Machado, F.R. (Ed.). Sepse. São Paulo, Atheneu, 13-20.

Stevenson, C.K., Kidney, B.A., Duke, T., Snead,
E. C. R., Mainar-Jaime, C. \& Jackson, M. L. 2007. Serial blood lactate concentrations in systemically ill dogs. Veterinary Clinical Pathology, 36(6):234-239.

Theobaldo, M.C. (2012). Efeitos da Solução Salina Hipertônica na Resposta Inflamatória na Sepse. Tese. (Doutorado em Ciências Médicas) - Pós-graduação em Ciências Médicas, Faculdade de Medicina da Universidade de São Paulo, São Paulo, f.94.

Thijs, L.G. \& Hack, C.E. (1995). Time course of cytokine levels in sepsis. Intensive Care Medicine, 21, 258 -263.

Vieira Júnior, J.M. (2014). Injúria renal aguda (IRA) relacionada à sepse. In.: Azevedo, L.C.P., Machado, F.R. (Ed.). Sepse. São Paulo, Atheneu, 13-20.

Von Dessauer, B., Bongain, J. \& Molina, V. (2011). Oxidative stress as a novel target in pediatric sepsis management. Journal Critical Care, 26 (3):103-107

\section{Recebido em Setembro 24, 2015}

Aceito em Novembro 2, 2015

License information: This is an open-access article distributed under the terms of the Creative Commons Attribution License, which permits unrestricted use, distribution, and reproduction in any medium, provided the original work is properly cited. 